\title{
The 'full-blown' MRI of sudden hearing loss: 3D FLAIR in a patient with bilateral metastases in the internal auditory canals
}

\author{
Giorgio Conte,${ }^{\bigotimes 1}$ Federica Di Berardino,${ }^{2}$ Diego Zanetti, ${ }^{2}$ Sabrina Avignone, ${ }^{1}$ Clara Sina,${ }^{1}$ Elisabetta lofrida, ${ }^{3}$ and \\ Fabio Triulzi ${ }^{1,4}$
}

${ }^{1}$ Neuroradiology Unit, Fondazione IRCCS Cà Granda Ospedale Maggiore Policlinico, Italy

${ }^{2}$ Audiology Unit, Fondazione IRCCS Cà Granda Ospedale Maggiore Policlinico, Italy

${ }^{3}$ Otolaryngology Unit, Fondazione IRCCS Cà Granda Ospedale Maggiore Policlinico, Italy

${ }^{4}$ Department of Pathophysiology and Transplantation, Università degli Studi di Milano, Italy

${ }^{\mathbb{C}}$ Corresponding author.

Giorgio Conte, Fondazione IRCCS Cà Granda Ospedale Maggiore Policlinico, U.O.C. di Neuroradiologia, Via

Francesco Sforza 35, 20122 Milano, Italy. Email: giorgioconte.unimed@gmail.com

Copyright $@$ The Author(s) 2017

\section{Abstract}

We report a case of a 57-year-old man with bilateral masses in the internal auditory canal. The peculiar findings at magnetic resonance imaging with tridimensional fluid-attenuated inversion recovery sequence combined with clinical data provided new insights into understanding the pathophysiology of the hearing loss.

Keywords: Magnetic resonance imaging, fluid-attenuated inversion recovery, inner ear, internal auditory canal, melanoma

\section{Introduction}

Different expansive lesions can involve the internal auditory canal (IAC) and the cerebello-pontine angle (CPA). Primary lesions include most frequently vestibular schwannomas and meningiomas; however, metastatic lesions have also been reported. ${ }^{1-4}$ Patients with expansive lesions of the IAC develop sensorineural hearing loss (SNHL); however, the pathophysiology of hearing loss associated with lesions of the IAC is unknown. It is usually attributed either to direct compression or infiltration of the cochlear branch of the VIII nerve, or to vascular compression in the IAC leading to peripheral ischaemia, or to indirect damage of the blood-labyrinth barrier within the cochlea.

We report a case of supposedly bilateral metastases in the IAC from skin melanoma in which the implementation of the magnetic resonance imaging (MRI) study with a three-dimensional (3D) fluidattenuated inversion recovery (FLAIR) sequence helped understanding the different pathophysiological mechanisms of SHNL in the two ears.

\section{Case report}

A 57-year-old man was evaluated in our department for cochlear implant candidacy in bilateral profound SNHL. There was no prior history of temporal bone fracture, meningitis, autoimmune disease or exposure to ototoxic drugs. Four years earlier, he had undergone excision of a melanoma of the skin in the lumbar region and a concurrent inguinal lymph node dissection, followed by prolonged treatment with monoclonal antibodies and checkpoint inhibitor immunotherapy. Up to 4 months before, he was considered free from 
disease. Two months prior to admission, the patient suffered a sudden profound SNHL in the right ear, associated with intense vertigo, not responsive to systemic corticosteroids. Six weeks later he complained of a rapidly progressive hearing loss in the left ear, and followed a few days later by a left facial paresis and a mild dysgeusia.

The audiometric testing confirmed a bilateral profound SNHL with complete anacusis in the right side and a pure-tone threshold average of $100 \mathrm{~dB} \mathrm{HL}$ at $500,1000,2000,4000 \mathrm{~Hz}$ in the left, with absent speech discrimination without lip reading. Otomicroscopy and tympanograms were normal on both sides. At clinical examination the patient had a facial paresis (House-Brackmann grade 3$)^{\frac{5}{}}$ and a mild ataxic marching. The remaining neurological evaluations were normal. Blood tests showed increased levels of: alanine aminotransferase $57 \mathrm{U} / \mathrm{I}$ (normal range 5-41); hyperglycaemia $164 \mathrm{mg} / \mathrm{dL}$ (normal range 70-110); neutrophils $89.2 \%$, within a normal white blood cell count. The remaining blood tests were normal. A bilateral hypoexcitability to caloric stimulation and by video-head-impulse test was observed. A promontory electrical stimulation test, which measures the capability of hearing electrical sounds by eliciting different electrical stimuli by a transtympanic needle placed on the promontory, was performed. This test predicts the electrical response of surviving spiral ganglion nerve fibre populations. $\underline{6}$ Our patient showed a very poor temporal and frequency discrimination, resulting in a very low residual functioning of both VIII cranial nerves.

MRI, performed using a 3 Tesla scanner (Achieva, Philips Healthcare, the Netherlands) showed bilaterally a mass of soft-tissue signal inside the IACs, protruding slightly into the CPAs but without mass effect on the brainstem (igure 1). The soft tissue was hyperintense on unenhanced gradient echo (GRE) T1weighted images (Figure 1(a)) and hypointense on 3D steady-state free procession T2-weighted compared to the cerebrospinal fluid (CSF) (iuure 1().); it enhanced after intravenous administration of contrast agent ( $0.1 \mathrm{ml} / \mathrm{kg}$ of Gadovist, Bayer Schering Pharma, Germany) on GRE T1-weighted sequence ( Figure 1(). ). The inner ear structures had normal morphology and no signal abnormalities of the

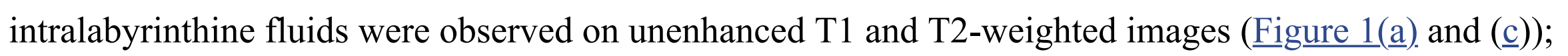
instead, unenhanced 3D FLAIR sequence with fat suppression showed hyperintensity of the right cochlea and vestibule, suggesting an increased protein content in the intralabyrinthine fluids, and the absence of hyperintensity in the left cochlea (Figure 1(마).). After the administration of contrast agent no enhancement was observed in the inner ear structures on GRE T1-weighted sequence (Figure 1(마)).

Considering the patient's history, MRI findings suggested the diagnosis of metastases of melanoma. The patient underwent CSF sampling through a lumbar puncture: the fluid was type B, clear, xanthochromic, with normal levels of glucose $(99 \mathrm{mg} / \mathrm{dL}$ ) (normal values $60-80 \%$ of serum level), raised CSF proteins $(133 \mathrm{mg} / \mathrm{dL})$ (normal values10-45) and mononuclear cells. A further MRI of the spine and whole-body positron emission tomography showed a disseminate meningeal metastatic disease, in the absence of other symptoms. The perspective of a cochlear implant was discarded and the patient was referred to the oncologist for consultation.

\section{Discussion}

We report the case of bilateral metastases of skin melanoma in the IAC, focusing on the distinctive features at MRI and on their relationship with the onset and progression of hearing loss.

The MRI findings of the inner ear in our case were peculiar: 3D FLAIR sequence showed a hyperintensity of the intralabyrinthine fluids within the cochlea and vestibule of the right ear, which suffered from abrupt, profound onset of deafness and vertigo. The hyperintensity on 3D FLAIR sequence is attributable to an increased protein content in the intralabyrinthine fluids. ${ }^{7,}, \underline{8}$ This assumption is in agreement with that reported by Silverstein and Schuknecht in 1966, who demonstrated that in patients with vestibular schwannoma the total perilymph protein was approximately nine times more than that in normal perilymph. The authors obtained a sample of inner ear fluids through a hole in the stapes footplate by using a micropipette. ${ }^{9}$ Although the anatomical differentiation of endolymph from perilymph has recently been demonstrated possible by acquiring a 3D FLAIR sequence at 4 hours after intravenous administration of contrast agent, $\frac{10}{-}$ the $3 \mathrm{D}$ FLAIR sequence can still not determine whether proteins increase in the 
perilymph or endolymph. Various explanations for increased intralabyrinthine protein content in vestibular schwannoma have been proposed: (a) blockage of neuroaxonal transport of proteins by tumour-related compression of the cochlear nerve; (b) cochlear membrane damage by arterial stasis resulting in increased permeability; and (c) cell-mediated immune reaction in the inner ear to antigenic components of vestibular scwhannomas. $\underline{6}$ The absence of high signal on T1-weighted images in the right inner ear excluded the presence of metahaemoglobin in relation to a subacute intralabyrinthine haemorrhage, but it did not rule out an older haemorrhage with the presence of haemoglobin in another phase of degradation. The absence of enhancement after contrast agent administration excluded the intralabyrinthine melanoma diffusion. Thus the combination of MRI sequences suggested acute right cochlear damage.

Conversely, on the left side, where the progression of the SNHL was gradual, no abnormalities of the intralabyrinthine signal were detected on MRI, possibly indicating a direct neoplastic infiltration of the cochlear nerve. This assumption is also supported by the clinical evolution of the disease: one week after the progression to profound hearing loss in the left ear, the patient also manifested an ipsilateral facial paresis.

To the best of our knowledge no previous studies have previously reported patients with bilateral pathological involvement of the IACs, but with different intralabyrinthine magnetic resonance signal patterns and corresponding audiological findings.

Unlike the radiological pattern of the inner ear that is really peculiar in this case, the IAC profile at MRI was very typical. Usually, bilateral IAC masses induce the assumption of neurofibromatosis type 2; they typically present as hysointense or hypointense on precontrast T1-weighted MRI. Conversely, bilateral melanoma metastases, which are very rare, 11 are characterised by hyperintense soft tissue masses within the IAC on precontrast T1-weighted MRI, as in our case. Furthermore, the clinical presentation was also different in age, onset and vestibular symptoms.

This case demonstrated that MRI with 3D FLAIR sequence for study of the inner ear, combined with the audiological examination, may provide new insights into the pathophysiology of the hearing loss in patients with masses of the auditory canal. In particular, it may help differentiate between direct infiltration of the cochlear nerve and indirect cochlear damage. Other studies on larger series are needed to substantiate our observation.

\section{Funding}

The authors received no financial support for the research, authorship, and/or publication of this article.

\section{Conflict of interest}

The authors declared no potential conflicts of interest with respect to the research, authorship, and/or publication of this article.

\section{References}

1. Gerganov VM, Hore N, Herold C, et al. Bilateral malignant melanoma metastases to the internal auditory canal/cerebellopontine angle: surgical management and preservation of function. J Neurosurg 2008; 108: 803-807. [PubMed: 18377262]

2. Brackmann DE, Doherty JK. CPA melanoma: diagnosis and management. Otol Neurotol 2007; 28: 529 537. [PubMed: 17414177]

3. Arriaga MA, Lo WW, Brackmann DE. Metastatic melanoma to the cerebellopontine angle. Clinical and imaging characteristics. Arch Otolaryngol Head Neck Surg 1995; 121: 1052-1056. [PubMed: 7646858]

4. Roditi RE, Kesarwani P, Barker EM, et al. Imaging case of the month: bilateral internal auditory canal melanoma. Otol Neurotol 2012; 33: e77-e78. [PMCID: PMC3845031] [PubMed: 22872181]

5. House JW, Brackmann DE. Facial nerve grading system. Otolaryngol Head Neck Surg 1985; 93: 146147. [PubMed: 3921901] 
6. Alfelasi M, Piron JP, Mathiolon C, et al. The transtympanic promontory stimulation test in patients with auditory deprivation: correlations with electrical dynamics of cochlear implant and speech perception. Eur Arch Otorhinolaryngol 2013; 270: 1809-1815. [PubMed: 23053370]

7. Bhadelia RA, Tedesco KL, Hwang S, et al. Increased cochlear fluid-attenuated inversion recovery signal in patients with vestibular schwannoma. AJNR Am J Neuroradiol 2008; 29: 720-723. [PubMed: 18238842]

8. Conte G, Di Berardino F, Sina C, et al. MR imaging in sudden hearing Loss, Time to talk. AJNR Am J Neuroradiol. Epub ahead of print 2017. DOI: https://doi.org/10.3174/ajnr.A5230 (accessed 25 May 2017).

9. Silverstein H, Schuknecht HF. Biochemical studies of inner ear fluid in man: changes in otosclerosis, Menière's disease, and acoustic neuroma. Arch Otolaryngol 1966; 84: 395-402. [PubMed: 5921712]

10. Sano R, Teranishi M, Yamazaki M, et al. Contrast enhancement of the inner ear in magnetic resonance images taken at 10 minutes or 4 hours after intravenous gadolinium injection. Acta Otolaryngol 2012; 132: 241-246. [PubMed: 22201230]

11. Chang MT, Michaelides EM. High rate of bilaterality in internal auditory canal metastases. Am J Otolaryngol 2015; 36: 798-804. [PubMed: 26545474]

\section{Figures and Tables}

\section{Figure 1.}

Open in a separate window

Precontrast gradient echo (GRE) T1-weighted image (a), three-dimensional (3D) fluid-attenuated inversion recovery (FLAIR) sequence (b), 3D steady state free procession sequence (c), post-contrast GRE T1-weighted image (d). Magnetic resonance imaging showed bilateral soft tissue masses (thin arrows) within the IACs, protruding slightly into the CPAs but without mass effect on the brainstem. The soft tissue was hyperintense on T1-weighted images and hypointense on T2-weighted images compared to the cerebrospinal fluid, with enhancement after contrast agent administration. 3D FLAIR sequence showed hyperintensity of the intralabyrinthine fluid of the right cochlea and vestibule (thick arrow). 